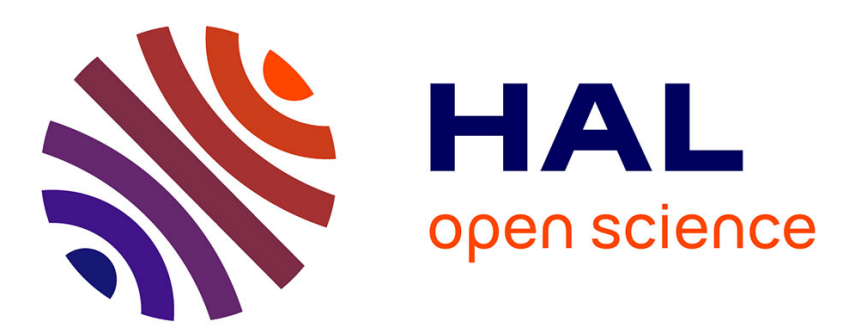

\title{
Direct illumination LED calibration for telescope photometry
}

E. Barrelet, C. Juramy

\section{To cite this version:}

E. Barrelet, C. Juramy. Direct illumination LED calibration for telescope photometry. Nuclear Instruments and Methods in Physics Research Section A: Accelerators, Spectrometers, Detectors and Associated Equipment, 2008, 585, pp.93-101. 10.1016/j.nima.2007.10.048 . in2p3-00188640

\section{HAL Id: in2p3-00188640 https://hal.in2p3.fr/in2p3-00188640}

Submitted on 19 Nov 2007

HAL is a multi-disciplinary open access archive for the deposit and dissemination of scientific research documents, whether they are published or not. The documents may come from teaching and research institutions in France or abroad, or from public or private research centers.
L'archive ouverte pluridisciplinaire HAL, est destinée au dépôt et à la diffusion de documents scientifiques de niveau recherche, publiés ou non, émanant des établissements d'enseignement et de recherche français ou étrangers, des laboratoires publics ou privés. 


\title{
Direct Illumination LED Calibration for Telescope Photometry
}

\author{
E. Barrelet \\ C. Juramy
}

November 1, 2007

\begin{abstract}
Laboratoire de Physique Nucléaire et de Hautes Énergies, CNRS - IN2P3 Universités Paris VI and Paris VII
\end{abstract}

Accepted by Nuclear Inst. and Methods in Physics Research, A NIMA 47931

Corresponding author: Etienne Barrelet

Address: LPNHE - Case 200, 4, place Jussieu, 75252 Paris Cedex 05, France

Tel. (+33)1 44272319 - Fax (+33)1 44274638 - barrelet@in2p3.fr

Keywords: photometry, telescope, calibration, LED, supernovae

\begin{abstract}
A calibration method for telescope photometry, based on the direct illumination of a telescope with a calibrated light source regrouping multiple LEDs, is proposed. Its purpose is to calibrate the instrument response. The main emphasis of the proposed method is the traceability of the calibration process and a continuous monitoring of the instrument in order to maintain a $0.2 \%$ accuracy over a period of years. Its specificity is to map finely the response of the telescope and its camera as a function of all light ray parameters. This feature is essential to implement a computer model of the instrument representing the variation of the overall light collection efficiency of each pixel for various filter configurations. We report on hardware developments done for SNDICE, the first application of this direct illumination calibration system which will be installed in Canada France Hawaii telescope (CFHT) for its leading supernova experiment (SNLS).
\end{abstract}

Classification codes: 95.55.-n, 95.55.Qf, 98.80.Es, 85.60.Jb 


\section{Introduction}

The next generation of cosmology experiments based on type Ia SuperNovae (SN Ia) light curves measurement, either in space or on ground, will have to control the systematic errors affecting the measurements of cosmic distances, roughly defined as the inverse square root of the number of photons received from these stellar 'standard candles'. The goal is to bring the systematic errors below the $1 \%$ RMS statistical error ${ }^{1}$ reached when averaging the many SN Ia events that will be measured in the next generation of experiments. We shall argue that, in addition to the classical calibration methods based on reference stars, this goal requires the development of new hardware calibration tools. This paper explores a photometric telescope calibration scheme aiming at a $0.2 \%$ accuracy over periods of years with a single measurement precision better than $0.1 \%$. It presents the requirements for a Calibrated Light Emitting Diode (CLED) source providing a direct illumination of the telescope. This CLED source should be composed of at least twenty high power LEDs covering the whole spectrum seen by CCD detectors. Such LEDs are becoming commercially available due to recent progresses of the technology. In addition this paper presents an overview of a complete calibration system, including a calibrated Cooled Large Area Photodiode (CLAP). The techniques are the same as those used in industrial photometric calibration systems. They are widely used in astronomy for detector test benches. The operation of an overall telescope calibration system integrating these elements within a wide field imager system will follow to a large extent that of high-energy physics calorimeter calibration systems [1].

In the present paper we focus on generic principles, presented hereafter. They will be driving our various objectives such as upgrading existing instruments or designing calibration systems for new ground and space-based telescope cameras. The applications will be discussed in following technical papers, beginning with the SNDICE proposal to equip the CFHT/Megaprime [2] currently running the SuperNova Legacy Survey (SNLS). We illustrate this paper with a few results of the R\&D done in view of SNDICE, which is presented in more details in [3].

\section{SN Ia Light Curve Measurement and Calibration}

We shall take the SNLS experiment as a model for the SN Ia photometric surveys performed to measure the expansion rate of the universe, to which we owe the introduction of vacuum energy ('dark energy') in the cosmological model. We

\footnotetext{
${ }^{1}$ For an average of 200 SN Ia per 0.1 redshift bin, taking a 15\% dispersion of supernovae intrinsic luminosity.
} 
shall expose in this paragraph the main features of the measurement method and the reasons for which new calibration devices are needed. We refer to a SNLS publication for details [4].

\subsection{Photometric measurements}

The first characteristic of the SNLS photometry, taking advantage of its large $1 \mathrm{deg}^{2}$ field of view, is to normalize the measurement of any supernova seen in a given frame to the average of a sample of reference stars seen in the same frame. This sample of more than 1200 main sequence stars per field is called the SNLS 'tertiary standard catalog'. Normalization yields a measurement of the flux of one point source relative to the average flux of many other point sources. Relative fluxes are not sensitive to the short term instabilities due to the atmospheric absorption (clouds,etc.), because they are essentially grey and uniform over the field of the camera. This hypothesis is consistently checked by SNLS for the tertiary standard stars, and bad frames are rejected. It is then assumed to hold for supernovae at various redshifts. Absolute fluxes, which are needed for the absolute calibration (cf. section 2.2), are affected by the fluctuations of both telescope and atmospheric transmissions. The former is measured by the system described in this paper, the latter is derived in SNLS by a statistical analysis which amounts to a selection of 'photometric nights' for which atmospheric transmission is peaked around a maximum value.

After emphasizing the usefulness of a large field of view for measuring fluxes, let us remark that non-uniformity is the price to pay. This problem has been quantified by SNLS [6]. First they make 'flat field' frames using twilight illumination in order to equalize the gain spread of the CCDs and the plate scale effect (4\%). Second they use dense stellar fields dithered within the field of view of Megacam camera to correct further its non-uniformity.

The stray light contribution to the flat fields is a suspected cause of the global $4 \%$ non-uniformity seen in red filters (10\% in blue and UV). After all corrections, residual non uniformities affecting flux ratios are still in a 2-4\% range and the color dependence of non-uniformity around $2 \%$ ( $1 \%$ for a 0.5 color ratio).

Contrary to twilight and stellar light sources, LEDs will map precisely filter transmissivity in a plurality of contiguous spectral bands. Non-uniformity is bound to increase in future telescopes, which plan to have larger fields of view and faster optics. LED illumination devices could bring them the information needed to control uniformity with a mass of data collected at any time of the day, independently of weather conditions. 


\subsection{Absolute calibration}

The second characteristic of the SNLS photometry is the 'absolute photometric calibration' of supernovae light curves by stellar photometry methods. This calibration is achieved through the following chain of comparison:

1. from 'primary' (Vega) to 'secondary' standard stars (from Landolt catalog[5]), using 'standard filters' (U,B, V,R,I)

2. from standard filters to Megacam filters ( $\left.u^{*}, g^{\prime}, r^{\prime}, i^{\prime}, z^{\prime}\right)$, using a set of secondary standard stars

3. from secondary standard stars to 'SNLS tertiary standard' stars, using Megacam filters

4. from 'aperture' to 'PSF' photometry estimators (respectively prefered for star calibration and for SNIa light curves), using tertiary standard stars

5. from tertiary standard stars to supernovae light curves in their rest frame, using SALT spectral model.

The operations from step (2) to step (4) use self-consistently the SNLS data taken within one Megacam field. Their global precision, known from the analysis reported in section 2.1, is around $1 \%$ in the g', r', i' bands. This number means that the dispersion of the luminosity measured with a given filter for an average main sequence star of a given magnitude and color would be around 1\% (RMS). This result is obtained without any description of Megacam filters. It just depends on the fact that the spectrum of a main sequence star is described by a single color parameter and therefore, when sampled in two different bands, it yields two parameters -luminosity and color- describing empirically the star. The accuracy, which contrarily to precision relates data from various experiments, depends on step (1) and includes all systematic effects affecting the flux measurement. It is not estimated directly.

\subsection{Spectral model}

Step (5), the last of the absolute photometric calibration process, extracts for each supernova a magnitude and a color parameter from the fluxes measured in two differents spectral bands at a time near to the peak luminosity. But contrarily to the previous calibration steps this one cannot be done by experimental means only. In order to deal with the effect of redshift, SNLS has to compare precisely the fluxes measured through different filters. Traditionally this spectral adaptation just consisted in computing two algebraic corrections (called S and K), but at the present 
level of precision, it requires the development of a numerical spectral model for all light sources and detectors.

SNLS has developped a software tool called SALT[7], which on one hand simulates the photometric response of Megacam to a given spectrum (either a standard star or a SN Ia) and on the other hand extracts a spectral model of SN Ia using available data (light curves and spectra). Let us present both aspects.

Firstly the spectral response of each Megacam filter is assumed to be determined by the product of one CCD quantum efficiency curve common to the whole focal plane and to one spectral response curve (both known from factory calibration). The absolute normalization of each spectral response is given by a coefficient fixing the 'zero point' magnitude, i.e. by forcing the photometric response to an ideal Vega source to be equal to 1. This modeling of Megacam is too crude. It will improve when, as mentioned in section 2.1 , it will include non-uniformities. However there are no experimental checks of the accuracy of the model. The calibration system proposed in this paper would allow to construct detailed maps of the instrument and to check their response to two dozens of narrow band LED sources calibrated with a better than $0.5 \%$ accuracy.

Secondly the SALT spectral model is fitted on the SN Ia data using a recursive algorithm. For each iteration the SALT spectra are computed and transformed according to a model describing both the specificity of a given supernova at a given phase and the type of instrument used. After that they are compared to the experimental data. As it is not possible to describe accurately all the instruments which have been used to observe supernovae, adhoc hypotheses are made under the form of adjustable photometric calibration factors and spectral correction curves. These adjustable parameters are needed to insure the compatibility of different data within a given spectral model. In particular the accuracy of SN Ia spectra found in databases is too bad to provide an estimate of the supernovae luminosities and of their color parameters. The color parameter is extracted instead from the light curve databases (as a ratio of fluxes measured in two adjacents bands). This color information is used to adjust the parameters describing either the empirical spectral correction, or the physical color of a given supernova.

The empirically corrected spectra are used to fit a SN Ia spectral model depending on two free parameters, one governing essentially the time dependence (sometimes called 'stretch') and the other the color ('B-V color'). For nearby supernovae the result of this fit does not depend on the spectral model since corrected spectra have been forced to reproduce photometric data, while for distant ones (or when using non standard filters) a good spectral model is necessary to yield good supernova parameters. In this context 'good' means 'in mean agreement with various published data', but not 'resulting of a traceable calibration process'. If calibrated light sources were commonly used, compiling data could become an exact science. 


\section{Direct Illumination LED Calibration Scheme}

\subsection{Principle}

In a direct illumination setup, photons propagate between the source and the detector in free space or through a telescope just as starlight does. Consequently the radiation field, besides the angular emission pattern of the source, depends only on geometrical factors and on the instrument transmission (neglecting multiple Fresnel reflection and diffraction effects). When known in one point, the field is known in every other point. This characteristic, combined with those of a LED, namely a point-like, stable, adjustable, bright source with an uniform angular distribution, makes a very attractive photometric calibration scheme. In the telescope calibration setup discussed hereafter, the source emission is restricted by baffling to the angular field of view of the telescope in order to suppress stray light. The striking characteristic of a LED compared to other sources is its 5-8\% FWHM spectral bandwidth. It has positive and negative consequences for our application. On one hand, one needs around 20 different LEDs to cover the spectral range of a CCD detector. This brings complexity in the control system and in the calibration procedure. But on the other hand, when using one LED at a time, the composite LED source offers a spectral granularity finer that the classical $U B V R I$ filter sequence by a factor 3 , free from the optical and mechanical problems related to the interposition of filters. The spectral distribution of a LED is easy to parametrize and smooth, thus avoiding aliasing and fringing problems when using an optical system having a sharp spectral response such as an interferometric filter.

\subsection{Critical features}

Selecting LEDs covering near UV to near IR range is not trivial. Many commercial off-the-shelf LEDs cannot be used because of their plastic dome structure transforming them into an ill-defined extended source. The best choice is the 'flat-top' geometry, where the light source is defined by the limits of the semiconductor dice (e.g. Agilent P505 green LED or OSRAM 'Golden Dragon' shown in Figure 1). In addition a minimal $0.1 \%$ LED efficiency ${ }^{2}$ is required in order to expose the focal plane detectors to a light flux comparable to the interesting astronomical sources (100 photon/pixel/s) for a maximum LED current $(50 \mathrm{~mA})$. Photodiodes able to monitor such low fluxes (pico-Amp currents) must be cooled and have a large area $\left(1 \mathrm{~cm}^{2}\right)$, hence the acronym CLAP. No standard photodiode calibration procedure is available at these low fluxes. However a photometric calibration procedure such as NIST's [9] can be applied to those CLAPs at a flux hundred times higher and

\footnotetext{
${ }^{2}$ Definitions of LED quantum efficiency are given in Appendix B.
} 

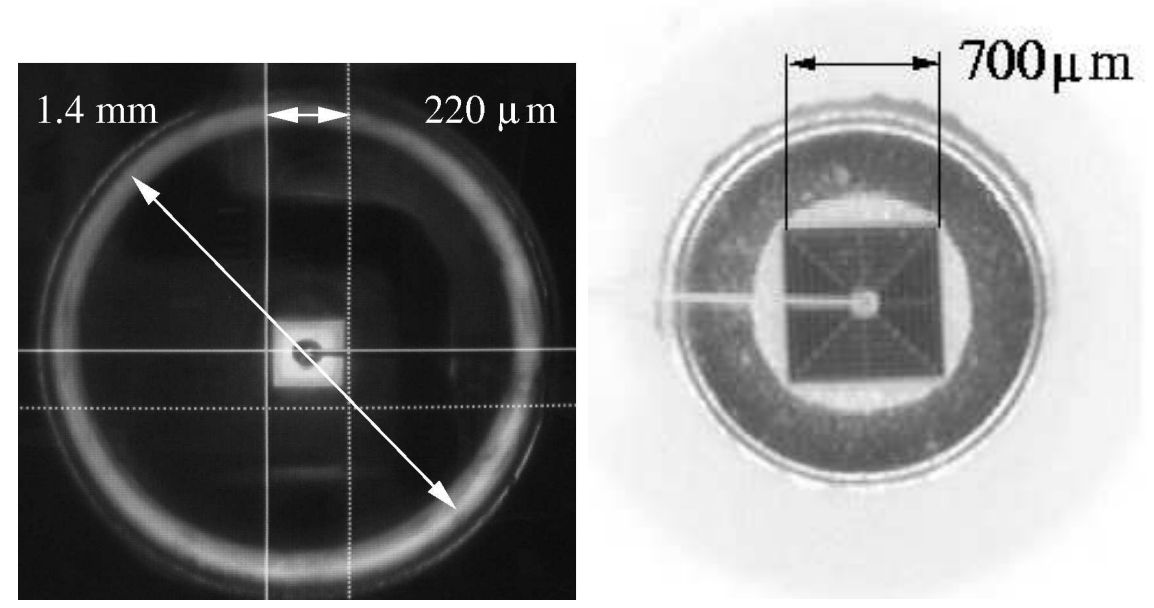

Figure 1: Flat-top LEDs. Left: Agilent P505, Right: OSRAM 'Golden Dragon'(respective quantum efficiencies $0.04 \%$ and $3.4 \% \mathrm{cf}$. Appendix B). Light emission is confined to central squares $(0.2 \times 0.2$ and $0.7 \times 0.7 \mathrm{~mm})$. Light ring around P505 $(\varnothing 1.4 \mathrm{~mm})$ is coming from diffusion on the cylindrical plastic wall. It has to be masked (see Figure 4).

then extended to very low fluxes by increasing the source-to-CLAP distance by a factor of ten (see section 3.3).

With recent progresses of LED technology, flat-top, high efficiency LEDs are becoming commercially available ('Golden Dragon' yields a $5 \%$ efficiency and a $400 \mathrm{~mA}$ maximum current). These high power LEDs allow a very flexible choice of exposure, by controlling electronically the duration and the radiant power of the illumination up to $10^{4}$ photon/pixel/s.

\subsection{The calibration setup}

The setup shown in Figure 2. a) is an optical bench used for the calibration of the CLED source and of the CLAP detectors. First it provides the 'Integral Radiometric Calibration' (defined in Appendix $\mathrm{A}^{3}$ ) of the CLED source using a calibrated photodiode traceable to NIST ${ }^{4}$ mounted on a $3 \mathrm{D}$ platform and read with a picoam-

\footnotetext{
${ }^{3}$ We have summarized in Appendix A and B the precise definition of radiometric and photometric quantities and their corresponding units, because they are not familiar to many physicists and not needed to follow our arguments.

${ }^{4}$ The National Institute of Standards and Technology provides calibrated photodiodes (type Hamamatsu S2281, $1 \mathrm{~cm}^{2}$ ).
} 

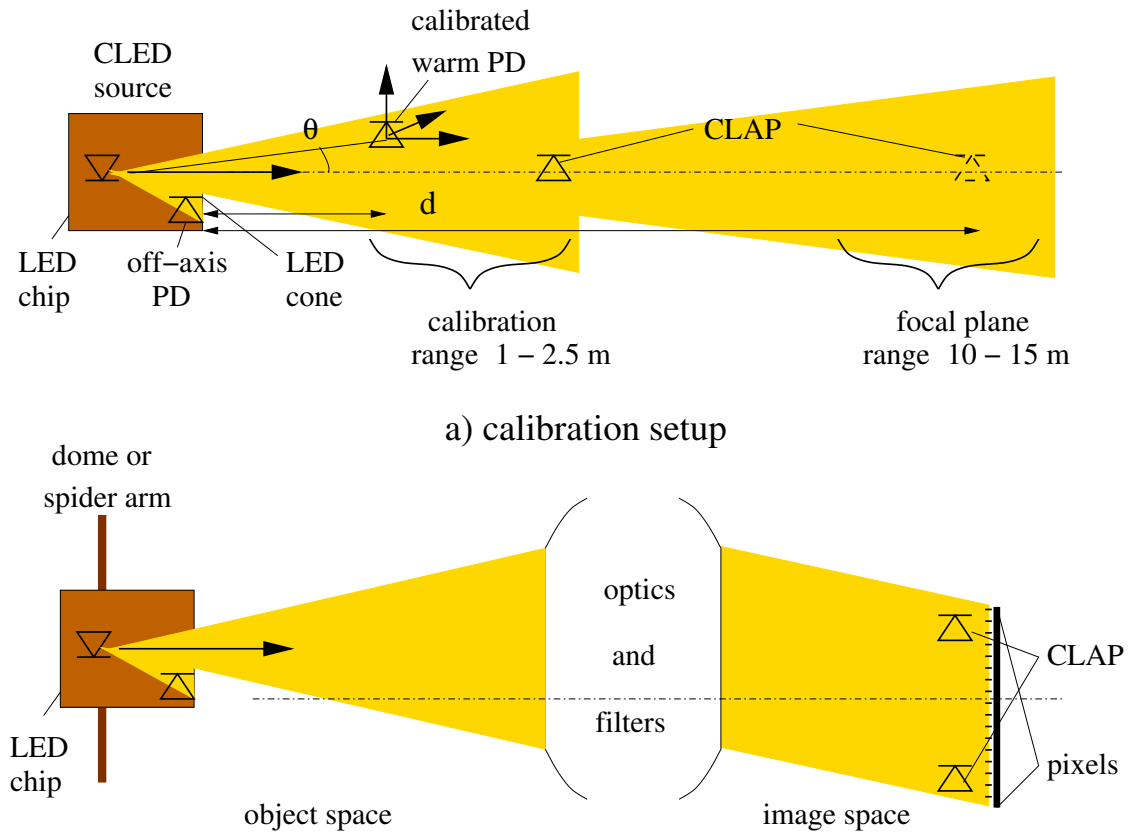

b) telescope setup

Figure 2: (a.) Setup for the calibration of LED light sources (CLED) and cooled large area photodiodes (CLAP). The calibrated PD maps the 3D radiation field in a $\theta=3^{\circ}$ cone. This yields the $2 \mathrm{D}$ radiant intensity map (W/sr) of the LED, which in turn yields the radiant power received on the CLAP. An extension of the optical bench to $15 \mathrm{~m}$ is needed to check the accuracy of CLAP read-out at low flux. (b.) Setup for the calibration of a full telescope and its camera (focal plane + optics + filters). 
meter. Secondly the CLAPs, alone or embedded in the focal plane, are calibrated using the CLED source around a one meter distance at 'high flux' (1 nA photocurrent). Then the distance between source and detector is increased to $\mathrm{d}=15 \mathrm{~m}$ in order to yield the 'low flux' CLAP calibration (10 pA photocurrent).

The setup drawn here for standalone CLAP detectors can be extended without any difficulty to the calibration of the large focal planes foreseen for the next generation of telescopes. The scope of the calibration would be extended using some CLAPs embedded in the focal plane. The resulting gain would be the possibility to calibrate directly the CCDs of the focal plane on the bench and, later, to monitor with the embedded CLAPs the efficiency of the transmission from the mirror to the focal plane, during the whole lifetime of the telescope. The related cost is to develop a system able to read out the CCDs and the CLAPs for a common CLED exposure. This feature is already included in the electronics presented in section 4 .

\subsection{The telescope setup}

Figure 2 b) represents the 'telescope setup' for monitoring a telescope camera, checking calibration accuracy and yielding photometric flat fields with programmable colors. The calibrated source is placed in the object space of the telescope, with its axis parallel to the instrument axis. In one version the source is fixed to a spider arm, in another to the dome structure (as represented in Figure 3). The signal received on the focal plane does not depend on the position of the source, except for the effects of mirror inhomogeneity ${ }^{5}$ and angle of incidence on optical surfaces or focal plane detectors. For a complete factory calibration of the telescope, the light source might scan the entrance pupil of the telescope in order to map the collection efficiency variations across the phase space. This is feasible in the dome version by moving the telescope relative to the source, while steering the axis of the source to keep it parallel to the telescope axis.

The spider version is the only one possible for a space telescope. It allows to calibrate the CLAPs imbedded in the focal plane on ground before launch, when CCDs do not work because they are warm. This calibration will be transported in space where it will be transferred to CCDs. Fixing duplicates of the LED source to other radial positions will assure redundancy and will calibrate the efficiencies at other angles of incidence.

During a SN Ia experiment, the CLED source controls regularly the photometric response of the focal plane detectors. It provides a 'check standard', as codified

\footnotetext{
${ }^{5}$ The shadow of dust grains is spread on thousands of channels. For small grains it is not seen or it is eliminated by the statistical processing of many exposures taken at different LED positions. Large grains on the primary mirror are more noxious, because they yield diffraction rings far from their geometrical shadow.
} 


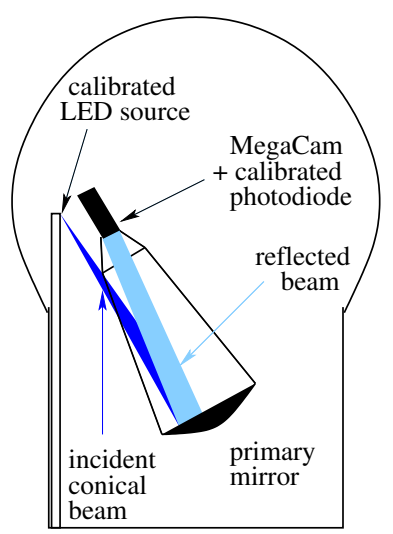

Figure 3: Dome setup for the SNLS experiment: the CLED source is at a fixed position inside the dome, the telescope is pointed towards the source for calibration exposure.

in [10]. Each 'calibration exposure' yields an ideal 'flat field' image where the respective response of pixels and CLAPs are proportional to their effective surface. The wavelength resolution of LEDs is ideal to monitor the color dependence of detectors and filters without being bothered by fringing effects. The fluctuations of the focal plane detectors and their electronics can be detected and corrected. Moreover monitoring the response of the CCDs illuminated by the calibrated light sources controls the long term stability of the telescope transmission. The calibrated source itself is monitored collectively by comparing the response of the CLAP and of the CCDs when illuminated by different LEDs. Each LED is monitored individually by its own photodiode (cf. section 4.1). The ultimate accuracy of the photometric measurements is determined by the long term stability analysis, obtained by comparing the results of the complementary, high precision, monitoring systems: we encounter here again the check-standard method. One finds an example of such an analysis in [11] where the collection efficiency of a detector (liquid argon) varies by $14 \%$ in 7 years, while thanks to a relevant check standard the accuracy of energy measurement is maintained within $0.3 \%$.

\section{Instrument Specifications}

These specifications were established for the hardware components developed in view of the SNDICE calibration of SNLS experiment[3]. 


\subsection{The LED Source}

This component is a block made of approximately 20 identical channels and a common electronic interface. Each channel is composed of a LED, a thermal sink, an optical baffling, an 'off-axis photodiode (PD)' for irradiance monitoring, two apertures defining a conical beam (a LED pinhole and an angular diaphragm). For the Agilent P505 LED seen in Figure 1, the minimal dimensions of this block are $10 \mathrm{~cm}$ in the direction of the optical axis and a $5 \mathrm{~cm}^{2}$ section in the plane perpendicular to it $\left(0.25 \mathrm{~cm}^{2}\right.$ per channel). Manufacturing is much easier if a larger section is allowed. Positioning precision for each LED relative to its angular aperture should be $\mathrm{O}(100 \mu \mathrm{m})$ in order to assure parallelism of the conical beam axes. The pinhole and angular apertures adapted to the same P505 LEDs would be respectively $200 \times 200 \mu \mathrm{m}^{2}$ and $\varnothing=3 \mathrm{~mm}$, assuming a $1.5^{\circ}$ angular aperture surrounded by a $0.1^{\circ}$ penumbra region. An extra channel with two pinhole apertures inferior or equal to the LED bright square creates a pencil beam for the alignment of source and telescope axes.

As an illustration of this concept, Figure 4 shows a model made in our laboratory including 6 LEDs which can be baffled one at a time by a movable mask. The measurement of the radiation field generated by this source has been measured as shown in Figure 5. The reproducibility of the field measurement is excellent ( $0.03 \%$ RMS), just limited by electronic noise, as seen in Figure 6.

For a precise angular alignment of the source and the telescope axes, an 'artificial planet' channel creates a parallel beam with a $\varnothing 10 \mu \mathrm{m}$ pinhole masking a LED and a $\emptyset 10 \mathrm{~mm}$ lens focussing it at infinity. It provides a 20 ” spot on the focal plane (which might also be used for the measurement of multiple Fresnel reflection effects showing up as weak large-angle halos).

The electronics proposed for controlling the LED source include a common computer interface, a temperature sensor, a programmable LED current controller (for addressing each LED and setting its illumination level and duration), LED current monitors and irradiance monitors. The irradiance monitors can be coupled to each LED, either by direct illumination ('off-axis photodiodes'), or by quartz fibers. The off-axis angle has to be small in order to sample the LED radiation field in the vicinity of the calibration field. For SNDICE each calibrated LED is monitored by a photodiode, which is mounted on a printed circuit board and adjacent to a circular hole through which the conical calibration beam passes (the mean off-axis angle is $3^{\circ}$ ). This pc-board is embedded in the beam defining body common to the 25 LEDs.

The LED controller generates and reads currents inside closed loops well above noise level. This 'current loop' characteristic is favorable for installing analog electronics at a distance from the light source, outside the telescope field of view 


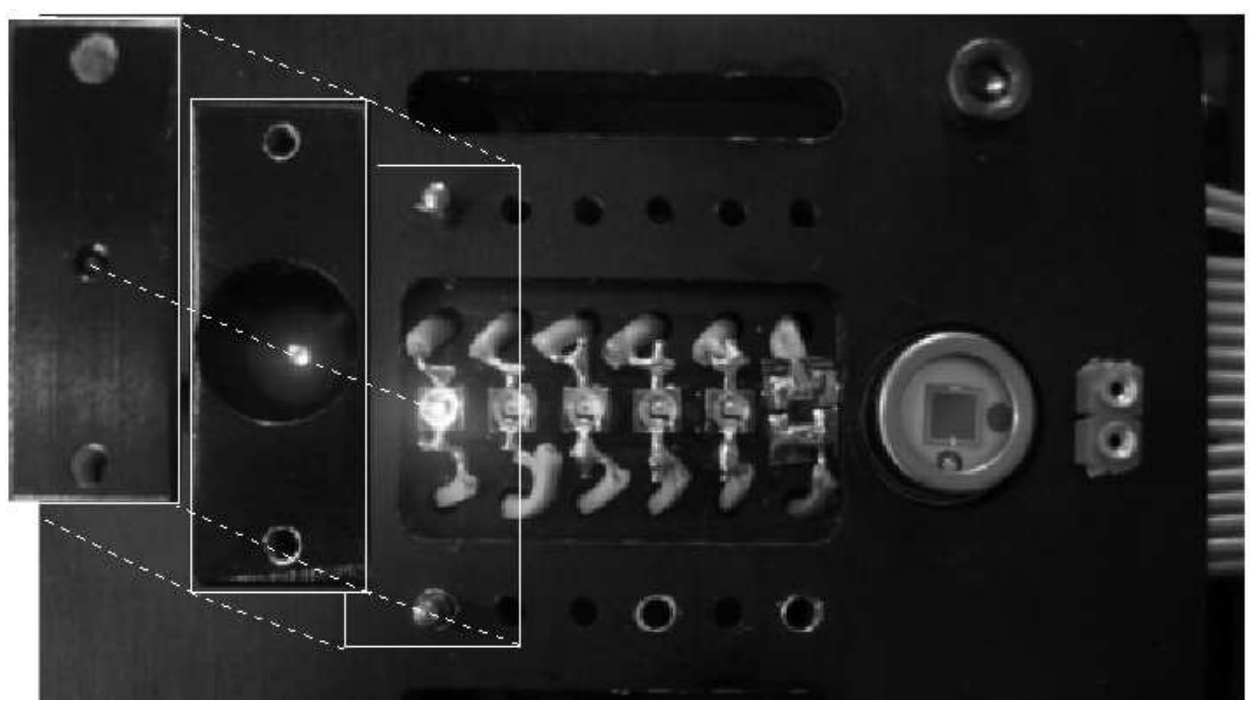

Figure 4: This light source model built at LPNHE/Paris is made of six LEDs on a base plate. The left most LED is lit. It is also seen on the left through a $\varnothing 0.5 \mathrm{~mm}$ hole drilled at the bottom of a 'low-mask' and through a $\varnothing 2 \mathrm{~mm}$ hole on the top of a 'high-mask'. Reflection on the edge of this thick hole yield a bump on beam profile (Figure 5). These three views are overlaid to suggest the mounting of the masks on the base plate. The photodiode seen on the right measures the light reflected on the optics and the detectors. 

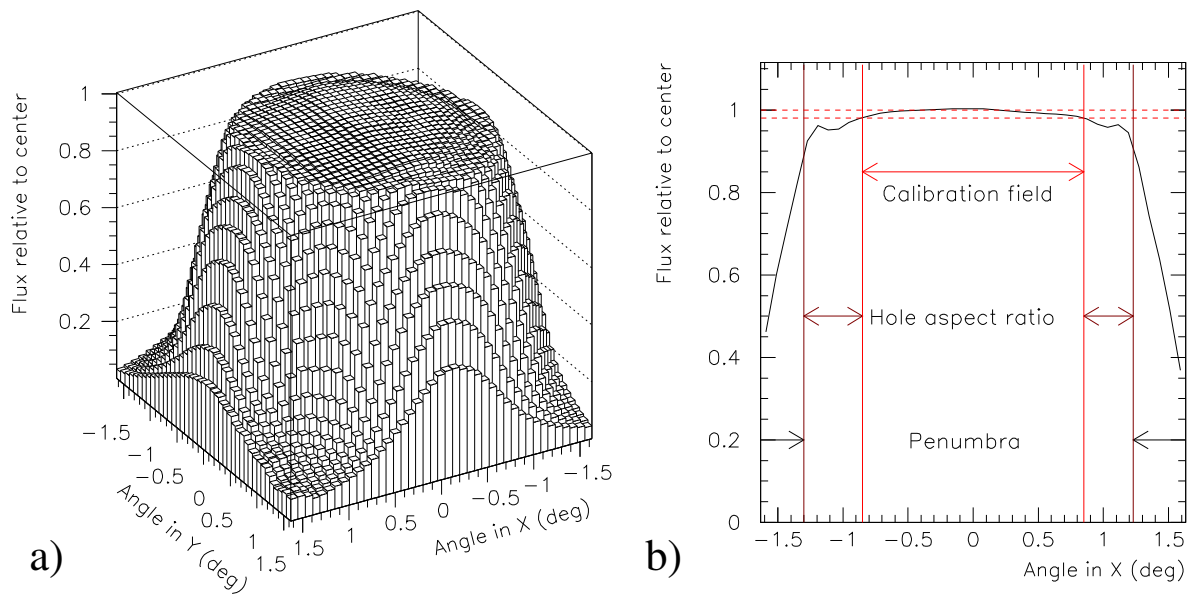

Figure 5: a.)XY scan of the illumination field of the LED light source in Figure 4. The photocurrent measured in the central flat field, generated by a $5.8 \mathrm{~mm}^{2}$ photodiode at $0.9 \mathrm{~m}$, is about $12 \mathrm{pA}$. b.) A cut of this field along a symmetry axis shows a $\pm 0.8^{\circ}$ calibration field with a $\pm 1 \%$ uniformity, surrounded by a rougher region of $0.4^{\circ}$ due to a bad aspect of the angular diaphragm and a penumbra zone.

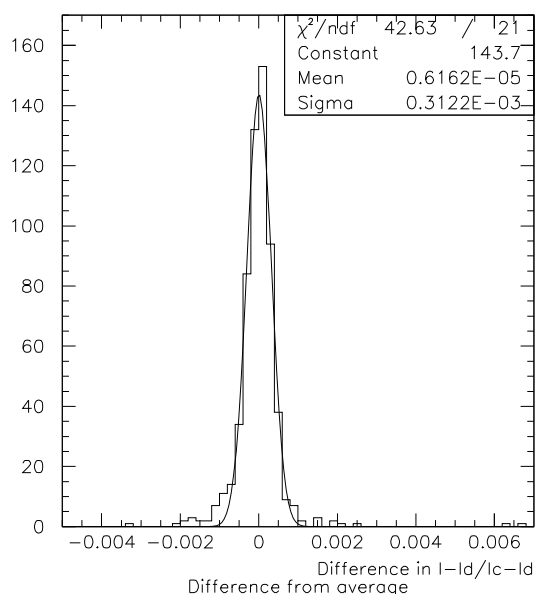

Figure 6: Reproducibility of the field map obtained by comparing 5 maps taken within 24 hours. The measure is limited by the noise level (4 fA rms for a signal of $10 \mathrm{pA})$. 
together with control and readout electronics. Another favorable characteristic is the possibility to cover a large range of radiant power by adjusting or by programming LED currents and to program different exposure durations (electronic and/or mechanical shutter). Table 1 summarizes the order of magnitude of the various photodiode currents $\mathrm{I}_{P D}$ to measure. Our controller system is very similar to several LED pulsing systems which have been built for optical calorimeters in high energy physics experiments. For example in H1-Spacal [12][13] the 405 LEDs illuminate 1600 photomultipliers and the 405 photodiodes, which provide each a measurement with a precision below $0.1 \%$.

\begin{tabular}{|c|c|c|c|c|c|c|}
\hline $\begin{array}{c}\text { Location } \\
\text { Distance }\end{array}$ & LED chip & $\begin{array}{c}\text { Monitoring } \\
\mathbf{4 c m}\end{array}$ & \multicolumn{2}{|c|}{$\begin{array}{c}\text { Calibration range } \\
\mathbf{1}-\mathbf{2 . 5 m}\end{array}$} & \multicolumn{2}{c|}{$\begin{array}{c}\text { Focal Plane } \\
\text { 15m }\end{array}$} \\
\hline detector type & & off-axis PD & calibrated PD & CLAP & CLAP & pixel \\
\hline detector size & $200 \mu \mathrm{m}$ & $4 \mathrm{~mm}$ & $5 \mathrm{~mm}$ & $1 \mathrm{~cm}$ & $1 \mathrm{~cm}$ & $15 \mu \mathrm{m}$ \\
\hline angular size & $1 \mathrm{rad}$ & $100 \mathrm{mrad}$ & $2-5 \mathrm{mrad}$ & $4-10 \mathrm{mrad}$ & $0.7 \mathrm{mrad}$ & $1 \mu \mathrm{rad}$ \\
\hline photon/s & $10^{14}$ & $10^{12}$ & $0.4-2.5 \times 10^{9}$ & $1.6-10 \times 10^{9}$ & $44 \times 10^{6}$ & 100 \\
\hline IPD (full scale) & $0.04 \% \times 40 \mathrm{~mA}$ & $160 \mathrm{nA}$ & $64-400 \mathrm{pA}$ & $0.26-1.6 \mathrm{nA}$ & $7 \mathrm{pA}$ & $0.016 \mathrm{fA}$ \\
\hline
\end{tabular}

Table 1: Photon counting rate and photocurrents with LED P505 at $40 \mathrm{~mA}$ (efficiency is $0.04 \%$ ).

For small systems, such as the test system of figure 4 or SNDICE, it is easy to achieve an electric stability better than a few $10^{-4}$ over a temperature range of a few degrees for the current generators and the monitoring equipment. For our test system the overall stability, electrical and optical, is kept at this level (figure 6). Besides, for SNDICE, the LEDs are kept in good thermal contact with a massive aluminium body and their temperature is monitored with a $0.1^{\circ} \mathrm{C}$ precision. The operation of this system will tell us at the $10^{-4}$ level (and will correct) the temperature dependency of the quantum efficiency and the spectrum of its LEDs. Concerning heat aging, what is known actually from datasheets is relevant only for harsh industrial applications averaging many thousands of hours at a high temperature (up to $150{ }^{\circ} \mathrm{C}$ ). Concerning radiation aging, it was checked that LED irradiance could keep its $0.3 \%$ accuracy during 10 years in a high radiation environment near a particle accelerator [14].

\subsection{The cooled photodiode}

The necessity to cool a CLAP in order to suppress dark current is justified by the small photo-current generated in the focal plane position, as found in Table 1. CLAPs can be mounted inside the camera's cryostat to share its cooling, or in a 
small vessel with a Thermo-Electric (T.E.) cooler and a temperature sensor (see Hamamatsu S3477 series). In the former case a Low Current Amplifier (LCA) adapted to the CLAP is the most convenient solution. This is the solution chosen for instance in the NIST amplifier for large area silicon photodiode described in [15]. It can be implemented with a commercial LCA with a few femto-Ampere input bias current (IBC), allowing to monitor the photocurrent waveforms of transient LED illumination. The practical problem is that we have found only one such LCA (LCA-30-1T, 10 fA IBC). Moreover with its fixed gain it does not cover the whole CLAP signal range. In the latter case, an off-the-shelf picoammeter (Keithley Model 617 \& 6514, 3 fA IBC) gives more versatility and performance.

In our test bench we have developed a system which cumulates the advantages of both apparatus. It uses a dual gain amplifier [16] connecting the analog output of Keithley 617 Programmable Electrometer to two 16 bits digitizers. This provides a system adding a $0.5 \mathrm{Mhz} / 21$ bits waveform digitizer to a Keithley preamplifier (low-IBC, low-noise, programmable gain, factory-calibrated). On this line we are developing an ASIC integrating an ultra-low IBC preamplifier with the dual gain amplifier and digitizer in order to equip several diodes on the same focal plane [17]. This ASIC has achieved a $1.3 \mathrm{~ms} / \sqrt{p A}$ and a $3 \mathrm{fA} / \sqrt{\mathrm{Hz}}$ resolution on single square light pulse (corresponding to a $0.07 \mathrm{e}^{-} / \mathrm{s} /$ pixel flux on the CCD).

We expect a $0.1 \%$ electrical accuracy and a precision $<0.1 \%$, with light-distance varying over a factor of 10 (current over a factor of 100). This corresponds to what is achieved with our current voltage measuring chain described in [18](chap.4), which uses a custom made dual gain amplifier. The irradiance versus distance relation offers a calibration of the pA range, in principle more accurate than Keithley's electrical calibration ( $2 \%$ accuracy).

\section{Conclusion}

Progress in LED technology offers high efficiency, point-source packaging and a complete wavelength coverage from near UV to IR. Although it is still difficult to conjugate all these qualities in one brand of LEDs, it is already possible, with quantum efficiency better than $0.1 \%$, to illuminate a telescope entrance aperture in order to calibrate photometrically a wide field camera.

In this paper we presented a light source covering near-UV to near-IR with 20 LEDs. The goal of its control and monitoring electronics is to guarantee a $0.2 \%$ stability and a precision better than $0.1 \%$. It will be calibrated on a classical photometric calibration bench traceable to NIST standard. A low-noise ASIC chain with a 21 bits dynamical range, a precision better than $0.1 \%$ and an electric calibration accuracy equal to $0.2 \%$ is being adapted to photodiode readout, as an alternative to 
commercial picoammeters. This system may be globally calibrated, by increasing the source-detector distance, down to an irradiance level two orders of magnitude lower than allowed by a classical photometric bench. This allows to use photodiodes in a focal plane as a check standard for the overall telescope calibration at a $0.3 \%$ level.

The direct illumination telescope calibration system based on these light source and detector components is well adapted to the requirements of light distance measurements for type Ia supernovae. Absolute photometric calibration of each pixel in a camera at a $0.3 \%$ level with a $7 \%$ FWHM spectral resolution is particularly attractive for controlling redshift induced biases. It is obvious that the system proposed here has to be completed with atmospheric transmission monitoring and checked against classical star calibration.

Beyond the scope of the SN Ia application, this calibration system has a generic merit which might interest other science fields, such as photometric determination of galactic redshifts in large surveys for weak lensing or baryon oscillation experiments. On technical ground, an 'absolute photometer' capability allows merging high accuracy data from various experiments, checking high precision modeling of the atmospheric transmission, recalibration of reference stars and improving the cosmological distance scale. For satellite missions it will enable the transfer of the $0.3 \%$ absolute photometric calibration from ground to space where the absence of atmospheric correction assure an identically good calibration of reference stars.

\section{Acknowledgments}

We would like to thank P. Astier, J. Guy, N. Regnault for introducing us patiently to the subtleties of supernova calibration and analysis, R. Pain, K. Schahmaneche for turning our ideas into reality, C. Veillet, the CFHT director, for encouraging this project, J-C. Cuillandre and the CFHT staff for numerous discussions. Last but not least we are indebted to our electronic, mechanical and computer groups at LPNHE which supported our project during the last years.

\section{Appendix A: Radiometric calibration constants}

The 'Integral Radiometric Calibration' of a light source assumes that its radiation field is radial. It yields a 'radiant intensity' map (W/sr), which transforms into an 'irradiance' map $E\left(\mathrm{~W} / \mathrm{m}^{2}\right)$ in the detector plane when multiplied by a geometrical 'inverse square distance' factor. For the geometry of Figure 2-a, this factor is $(\cos \theta)^{3} / d^{2}$, where $d$ is the distance from the source to the detector plane and $\theta$ the angle between a light ray and the plane. For Figure 2-b, it is the 'plate scale' factor 


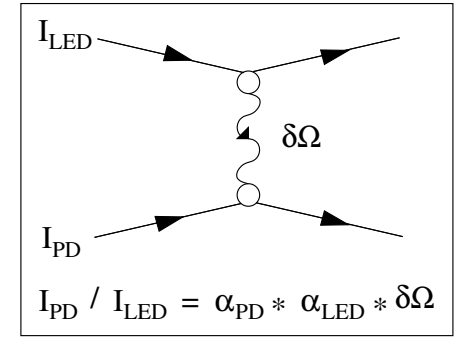

Figure 7: LED and photodiode quantum efficiencies.

(1/ $F^{2}$ where $F$ is the effective focal length of the telescope). The instant response of a photodiode is a photoelectric current $J$ characterized by the 'Radiant Power Sensitivity' $J / F(\mathrm{~A} / \mathrm{W})$, where $\Phi=E * A$ is the radiant power on a detector of surface area $\mathrm{A}\left(\mathrm{m}^{2}\right)$. This definition supposes that the readout electronics is equivalent to a calibrated ammeter and that the irradiance and the light collection area are known precisely.

For imaging detectors the area of a pixel is not known individually with the required accuracy, but it can be assumed on average, supposing a regular paving of the imager. A pixel charge sensing electronics cannot be calibrated individually ${ }^{6}$. Therefore, the imager's electric sensitivity is expressed in ADC units per exposure time (ADU/s) and the calibration constant for each pixel, i.e. the radiant power sensitivity is expressed in (ADU/W.s). In order to study deeper the behavior of the imager's pixels (quantum efficiency, gain, area), a detector model is needed. For instance a model using the same quantum efficiency and same electronic gain for all pixels is adequate for CCDs but would not be applicable to active pixel imagers.

By definition integral radiometric quantities are integrated on the spectral distribution of a specific light source within a wavelength interval $\Delta \lambda$. To each 'integral' quantity corresponds a 'spectral' one, for example the 'spectral radiant intensity' $I(\lambda)=\delta I / \delta \lambda(\mathrm{W} / \mathrm{sr} / \mathrm{nm})$. LED spectra being rather narrow $(\Delta \lambda / \lambda=5-8 \%$ FWHM), the factorization of the spectral dependence of the radiation field over phase space is a good approximation.

\section{Appendix B: Quantum Efficiency (Q.E.)}

Knowing the average energy of LED photons $\langle\mathrm{h} \nu\rangle=\mathrm{e}\langle\mathrm{V}\rangle$, one can transform the calibration constants of LED and photodiodes into effective quantum efficiencies.

\footnotetext{
${ }^{6}$ Absolute gain is deduced from the photoelectric shot noise ('Poisson' statistics).
} 
First multiply the radiant power sensitivity $(\mathrm{A} / \mathrm{W})$ by $\langle\mathrm{V}\rangle$ in order to express the PD calibration constant as the average quantum efficiency for LED's photons $\alpha_{P D}$ (\%). Then divide the radiant intensity (W/sr) of a LED by $\mathrm{I}_{L E D} * \mathrm{~V}$ in order to express the LED calibration constant as a quantum efficiency $\alpha_{L E D}(\% / \mathrm{sr})$ for photon emission in the solid angle $\delta \Omega$. The macroscopic quantity which reflects quantum statistics is the ratio of the currents flowing in a photodiode and in a LED is $\mathrm{I}_{P D} / \mathrm{I}_{L E D}$ (symbolized by this graph). The probabilities that an electron crossing a LED gap recombines to give a photon and that a photon is converted in a electronhole pair in the photodiode are near to 1 . Therefore $\alpha_{L E D}$ and $\alpha_{P D}$ are essentially the probabilities that a photon goes from LED to air and from air to photodiode. The fact that $\alpha_{L E D} \ll \alpha_{P D}$ reflects that it is more difficult for light to get from a high to a low refraction index medium than the contrary.

For a numerical application, let us take the specifications of the P505 LED in Figure 1:

$6.3 \mathrm{mcd} / \mathrm{sr}=6.3 / 683 \approx 9.2 \mathrm{~mW} / \mathrm{sr}$ at $\mathrm{I}_{L E D}=10 \mathrm{~mA} /<\lambda>=0.569 \mu \mathrm{m}$ (photon energy $2.18 \mathrm{eV}$ ).

The number of photons per steradian emitted by an electron in a $\delta \Omega$ solid angle around $0^{\circ}$ is:

$$
\alpha_{L E D}=9.2(\mathrm{~mW} / \mathrm{sr}) / 10(\mathrm{~mA}) / 2.18(\mathrm{eV})=0.042(\% / \mathrm{sr})
$$

The total Q.E. is obtained by integrating the LED emission over solid angle. It corresponds to an effective solid angle $\Omega_{t o t}$, equal to $2 \pi, \pi$, $<\pi$, respectively for hemispherical, Lambertian, parabolic emission patterns. Taking $\Omega_{t o t}=\pi$, Q.E. is $\alpha_{L E D}^{t o t}=0.13 \%$. The radiometric efficiency (W/W) is almost equal to Q.E. (photon/electron), considering that the mean voltage drop through the LED is about the mean photon energy. The photometric efficiency (lumen/W), usually quoted by industry, is proportional to the radiometric efficiency, the lumen/W constant being $1 / 683$ at $\lambda=0.555 \mu \mathrm{m}$. For the P505 LED example efficiency is $0.89 \mathrm{~lm} / \mathrm{W}, 10$ to 50 times smaller than a state-of-the-art efficient LED. We present in Table 2 how to get from current measurements to the LED efficiencies, for two types of LEDs mentioned in this paper.

\section{References}

[1] J.W.Mitchell et al., Nucl.Phys. B (Proc. Suppl.) 32 (1993) 106-114

[2] O.Boulade et al., SPIE 4841 (2003) 72-81

[3] K.Schahmaneche, LPNHE 2006-07 


\begin{tabular}{l|c|c|} 
& Agilent LED & OSRAM LED \\
\hline Measured current in LED (near full scale) & $35.5 \mathrm{~mA}$ & $300 \mathrm{~mA}$ \\
\hline Measured current in $5.7 \mathrm{~mm}^{2}$ PD at $90 \mathrm{~cm}$ & $25.4 \mathrm{pA}$ & $19.2 \mathrm{nA}$ \\
\hline Central wavelength $\lambda_{\max }$ & $600 \mathrm{~nm}$ & $590 \mathrm{~nm}$ \\
\hline Radiant power sensitivity @ $\lambda_{\max }$ & $408 \mathrm{~mA} / \mathrm{W}$ & $400 \mathrm{~mA} / \mathrm{W}$ \\
\hline Photodiode QE at $\lambda_{\max }\left(\alpha_{P D}\right)$ & $84.4 \%$ & $84.2 \%$ \\
\hline Irradiance at $90 \mathrm{~cm}$ & $10.9 \mu \mathrm{W} / \mathrm{m}^{2}$ & $8.42 \mathrm{~mW} / \mathrm{m}^{2}$ \\
\hline Radiant intensity & $8.83 \mu \mathrm{W} / \mathrm{sr}$ & $6.82 \mathrm{~mW} / \mathrm{sr}$ \\
\hline Total emitted power $\left(\Omega_{t o t}=\pi\right)$ & $27.7 \mu \mathrm{W}$ & $21.4 \mathrm{~mW}$ \\
\hline Available power from current & $73.3 \mathrm{~mW}$ & $619 \mathrm{~mW}$ \\
\hline Emission efficiency $\left(\alpha_{L E D}\right)$ & $\mathbf{0 . 0 3 8 \%}$ & $\mathbf{3 . 4 0 \%}$ \\
\hline Number of emitted photons $/ \mathrm{s}$ & $8.4010^{13}$ & $6.3610^{16}$ \\
\hline Photocurrent on $33.6 \mathrm{~mm}{ }^{2}$ PD at $10 \mathrm{~cm}(\mathrm{same} \mathrm{QE})$ & $11.7 \mathrm{nA}$ & $8.86 \mu \mathrm{A}$ \\
\hline Photocurrent on $1 \mathrm{~cm}^{2} \mathrm{PD}$ at $13.5 \mathrm{~m}(\mathrm{same} \mathrm{QE})$ & $1.98 \mathrm{pA}$ & $1.50 \mathrm{nA}$ \\
\hline Photons/s/pixel $($ size $13.5 \mu \mathrm{m})$ at $13.5 \mathrm{~m}$ & 27 & 20200 \\
\hline
\end{tabular}

Table 2: Comparison from measurements between an Agilent LED and an OSRAM Golden Dragon LED (LY W57B), using the quantities defined in Appendix A and B. The 'Golden Dragon' has a larger emitting surface (see Figure 1) and is more efficient.

[4] P.Astier et al., A\&A 447 (2006) 31

[5] A.U.Landolt, AJ 112 (1992) 340

[6] N.Regnault, astro-ph/0610397, to be published in ASP Conf. Ser., Vol.999 (2007)

[7] J.Guy et al., A\&A 443 (2005) 781

[8] C.W.Stubbs et al., astro-ph/0609260 (2006)

[9] G.P.Eppeldauer, NIST technical Note 1438 (2001)

[10] NIST/SEMATECH e-Handbook of Statistical Methods, http://www.itl.nist.gov/div898/handbook/index.htm

[11] E.Barrelet et al., Nucl. Instr. and Meth. A 490 (2002) 204

[12] R.D. Appuhn et al., Nucl. Instr. Meth. A426(1999) 518-537

[13] J.Janoth, Thesis, Heidelberg (1996)

[14] J.Ferencei, Private Communication (2006) 
[15] G.Eppeldauer and J.E.Hardis, Applied Optics Vol.30, No.22 (1991)

[16] E.Barrelet, C.Juramy, H.Lebbolo, R.Sefri, LPNHE 2004-11

[17] E.Barrelet, H.Lebbolo, R.Sefri, LPNHE 2007-04

[18] C.Juramy, Thesis, Paris 6 (2006) 